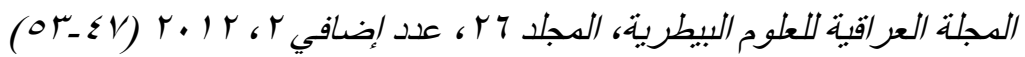

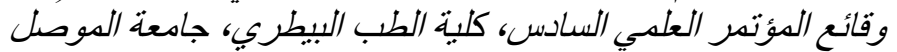

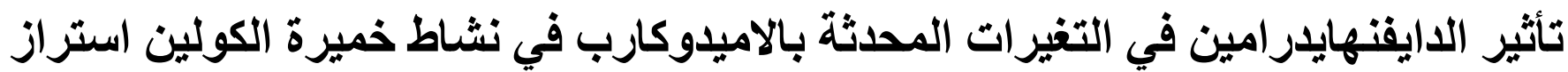

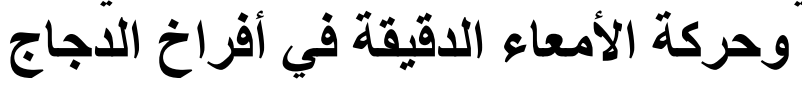 \\ ندى خليل إبراهيم وغادة عبد المنعم فارس
}

فرع الفسلجة والكيمياء الحياتية و الأدوية،كلية الطب البيطري، جامعة الموصل، الموصل،العراق

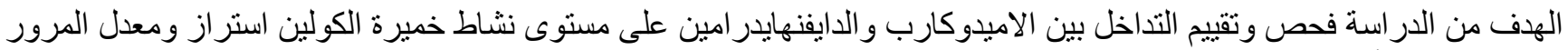

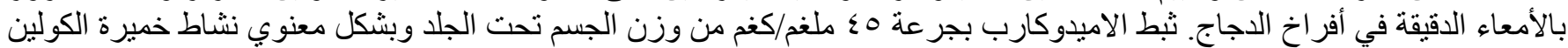

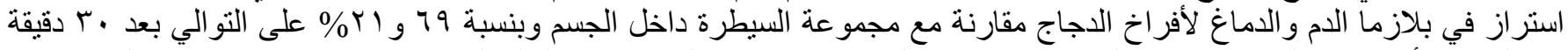

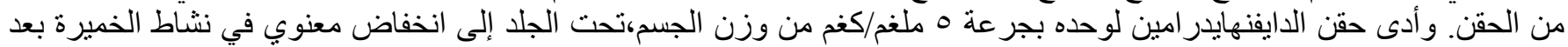

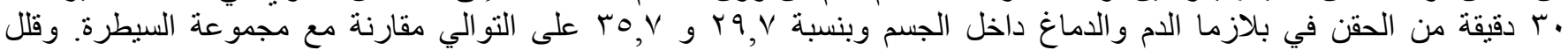

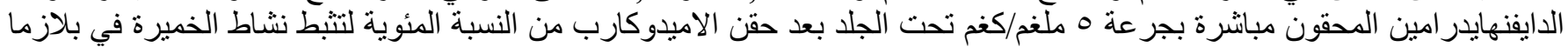

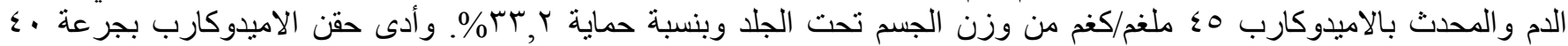

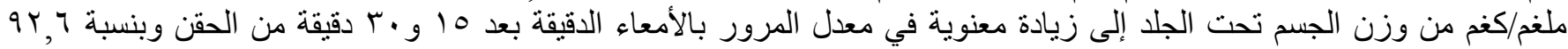

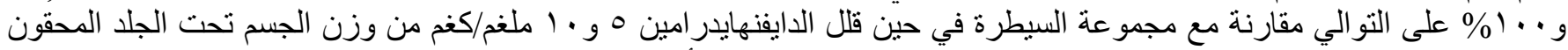

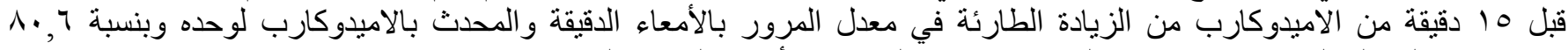

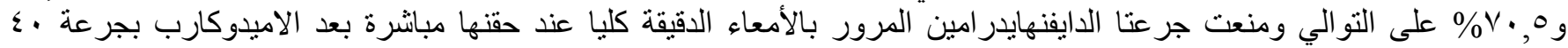

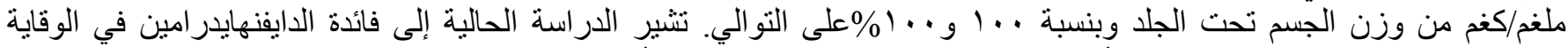

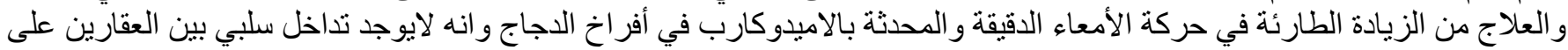

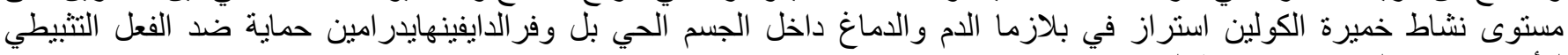
للأميدوكارب على مستوى نشاط الخميرة.

\section{Effect of diphenhydramine on the changes in cholinesterase activity and intestinal motility induced by imidocarb in chicks}

\author{
N.K. Ibrahim and G.A. Faris
}

Department of Physiology Biochemistry and Pharmacology, College of Veterinary Medicine, University of Mosul, Mosul, Iraq

\begin{abstract}
This study examines and evaluates the interaction between imidocarb and diphenhydramine on the level of cholinesterase activity and small intestinal transit (SIT) in chicks. Imidocarb at $45 \mathrm{mg} / \mathrm{kg}$,s.c. significantly inhibited plasma and brain cholinesterases, $30 \mathrm{~min}$ after injection by 69 and $21 \%$, respectively. Diphenhydramine at $5 \mathrm{mg} / \mathrm{kg}$, s.c significantly inhibited plasma and brain cholinesterase by 29.7 and $35.7 \%$, respectively and significantly decreased the inhibitory effect of imidocarb on plasma cholinesterase by $33.2 \%$. Imidocarb at $40 \mathrm{mg} / \mathrm{kg}$, s.c. significantly increased SIT 15 and 30 min after injection by $92,100 \%$, respectively. Diphenhydramine at 5 and $10 \mathrm{mg} / \mathrm{kg}$, s.c., $15 \mathrm{~min}$ before imedocarb at $40 \mathrm{mg} / \mathrm{kg}$, s.c. significantly decreased SIT produced by imidocarb alone by 80.6 and $70.5 \%$, respectively and prevented the SIT when given immediately after imedocarb at $40 \mathrm{mg} / \mathrm{kg}$, s.c. by $100 \%$. The data revealed that diphenhydramine has a beneficial effect in controlling the SIT modulated by imidocarb in chicks and there was no adverse interaction between two drugs at the level of cholinesterase.
\end{abstract}

Available online at http://www.vetmedmosul.org/ijvs 


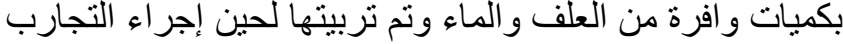

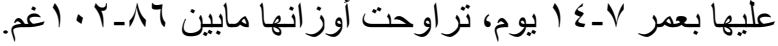

تحضير الأدوية للحقن

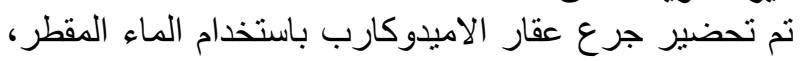

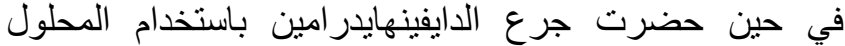

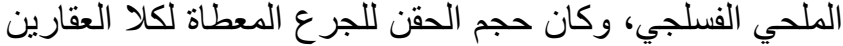
ه مل/كغ من وزن الجسم، تحت الجلد.

\section{قياس نشاط خميرة الكولين استراز}

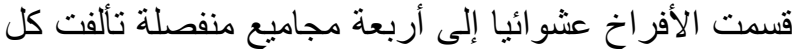

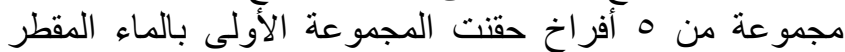

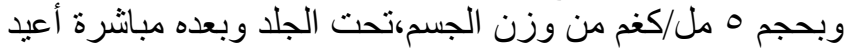
حقنها بالمحلول الملحي الفسلجي (شركة النصر الندر للكيمياويات

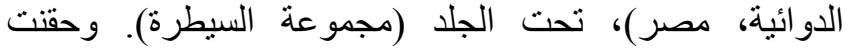

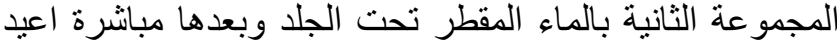

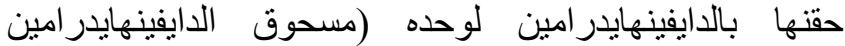
هايدروكلورايد، الثركة العامة للأدوية والمستلزمات لامنات الطبية،

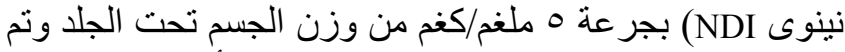
التوصل إلى هذه الجرعة اعتمادا على تجارب إن أولية حيث الجن كانت

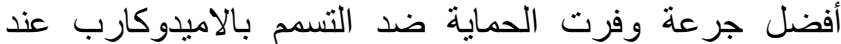

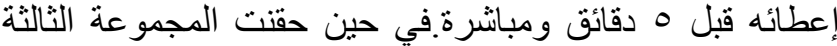

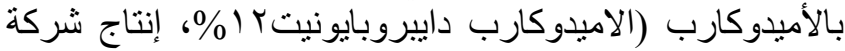
Saudi pharmaceutical industries بجرعة 0ـ ملغم/كغم من وزن الجسم تحت الجلا بعدها مباشرة

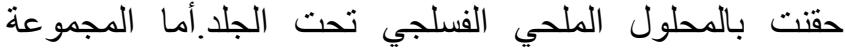

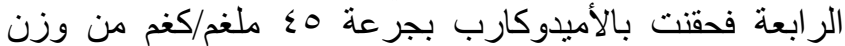

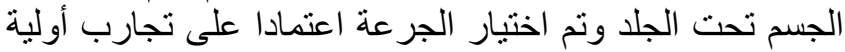

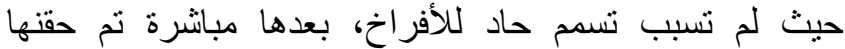
بالدايفينهايدر امين بجرعة ه ملغم/كغم من وزن الجسم تحت

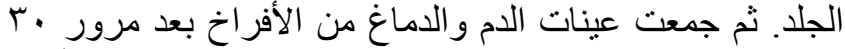

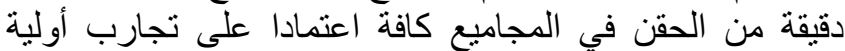

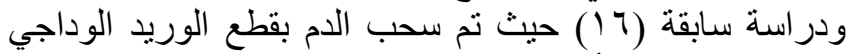

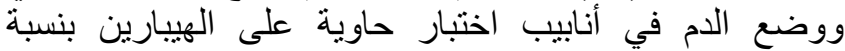

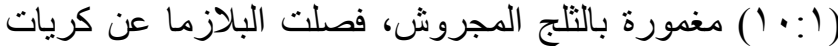

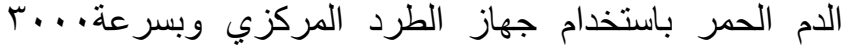

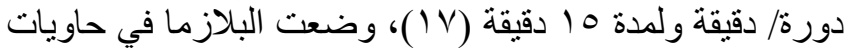

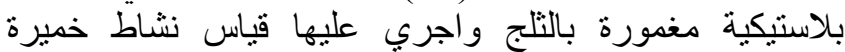
الكولين استراز مباثرة وقتلت الحيو انات بخلع الرقبة واستخر اج

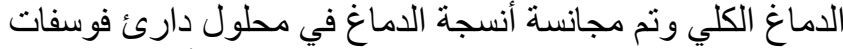

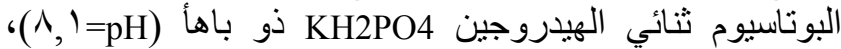

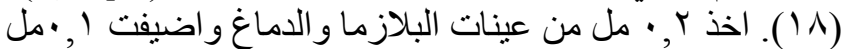

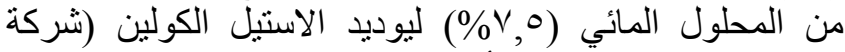

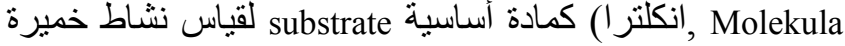
الكولين استراز بالطريقة الكهرومترية المحورة (9 (1).

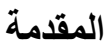

dipropionate) (Imidocarb يعد الاميدوكارب داييروبايونيت

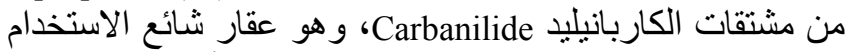

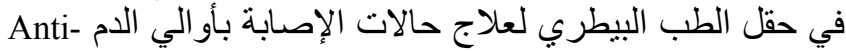

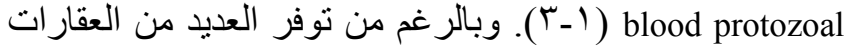

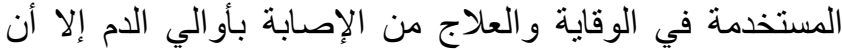

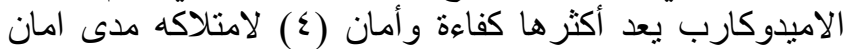

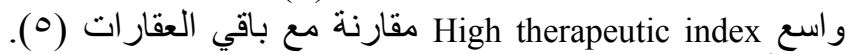
غير أن فعاليته العلاجية غالبا ما تكون مصحوبة بظهور بالية آثار

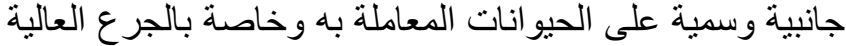

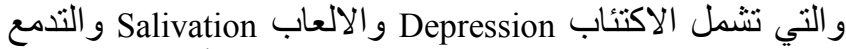
Lacrimation

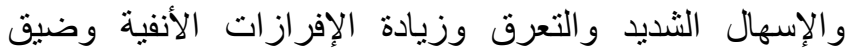

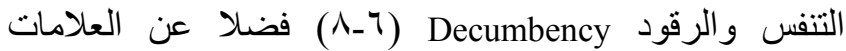

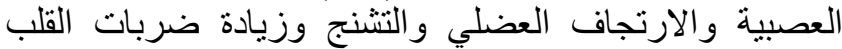

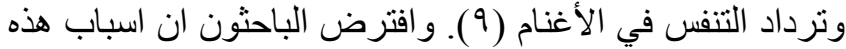

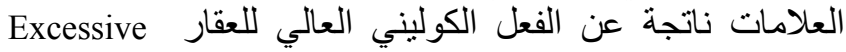

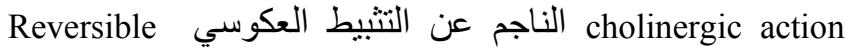
inhibition

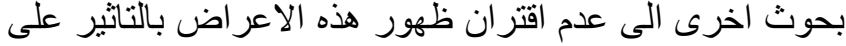

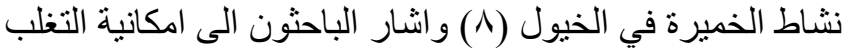

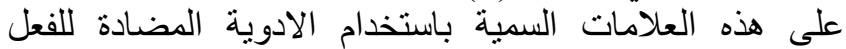

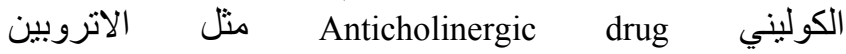
و الكلايكوبايروليت Glycopyrrolate (r (1). ولكون الاتروبين

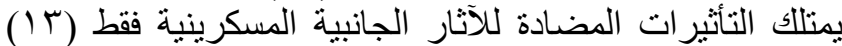

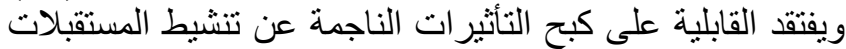

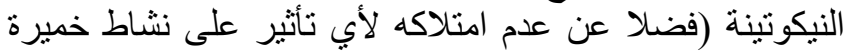

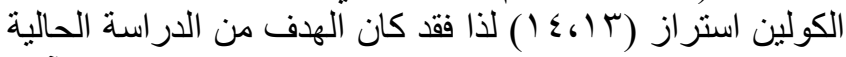

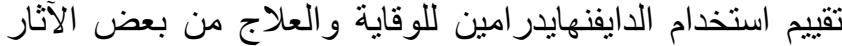

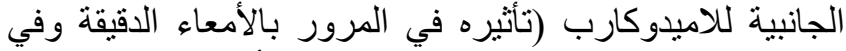

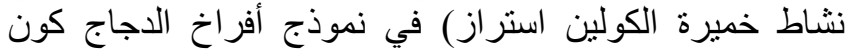

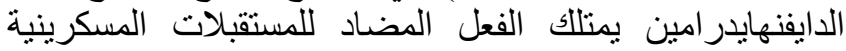

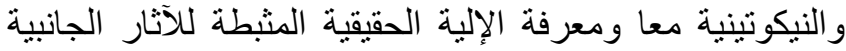

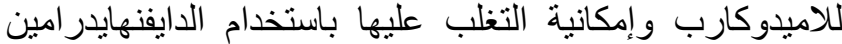

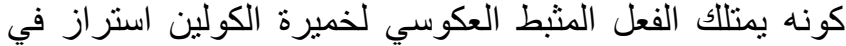
بلازما دم ودماغ أفر اخ الاجاج (10)

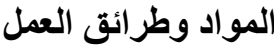

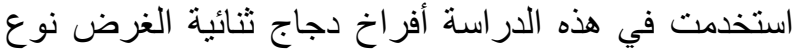

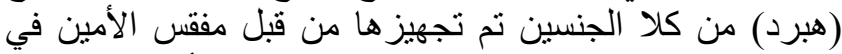

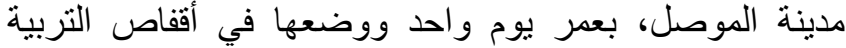

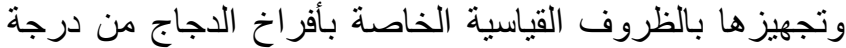

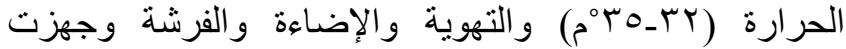


وزن الجسم تحت الجلد وبعده مباشرة أعيد حقنها بالمحلول

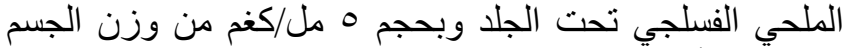
وحقتت الأفراخ في المجموعة الثانية والثالثة بالاميدوكارب

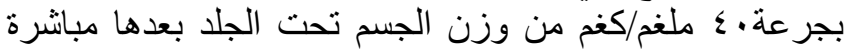

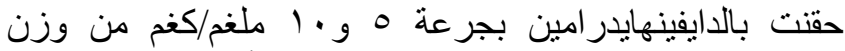

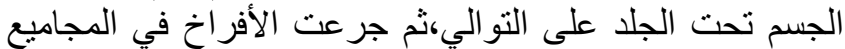

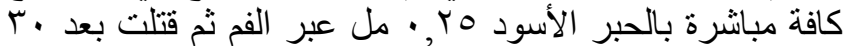

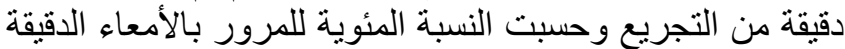
فضلا عن حساب النسبة المئوية لتثبيط المرور بالأمعاء الدقيقة الأفعاء الأية

$$
\text { وكما ذكر سابقا. }
$$

التأثير الوقائي للايفينهايدرامين عند إعطاءه قبل 10 دقيقة من

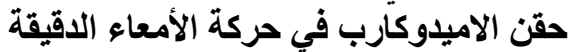

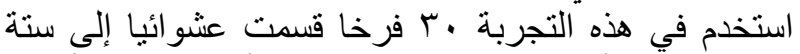

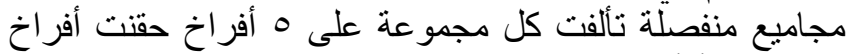

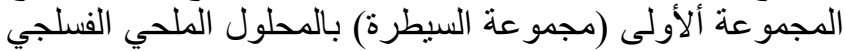

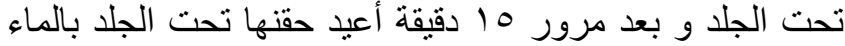

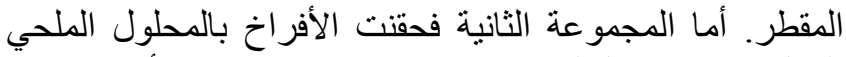

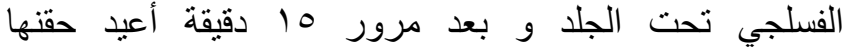

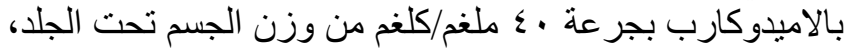

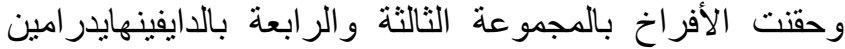

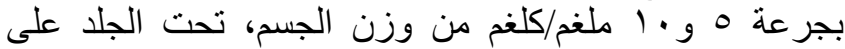

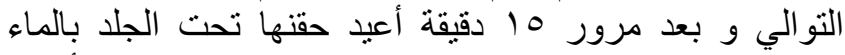

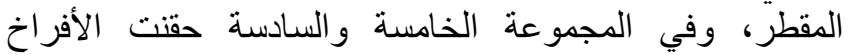

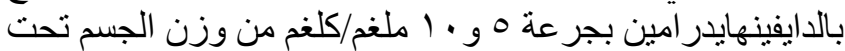

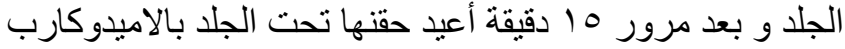

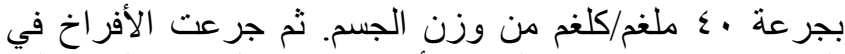

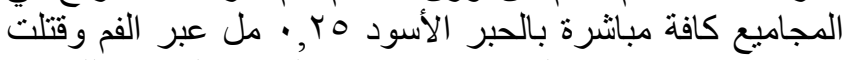

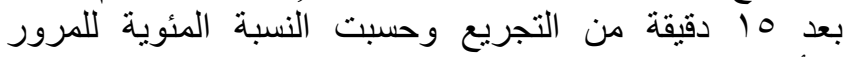
بالأمعاء الدقيقة فضلا عن حساب النسبة المئوية لتثبيط المرور

بالأمعاء الدقيقة وكما ذكر سابقا.

one way التلت النتائج إحصائيا باستخدام تحليل التباين analysis of variances

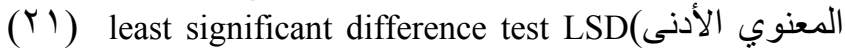
وكان مستوى الاختلاف المعنوي عند مستوى احتمالية

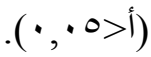

النتائج

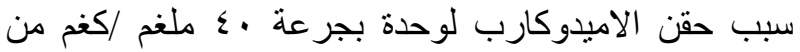

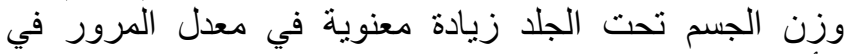

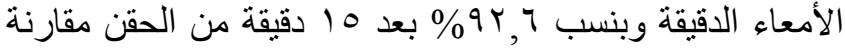

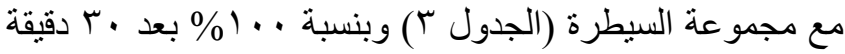

قياس المرور بالأمعاء الدقيقة (SIT) يقيس هذا الاختبار حركة الأمعاء الاقيقة GIT ونسبة الماء المرور فيها والتي تكون حساسة للأدوية المؤثرة في الجهاز الأنة الكوليني

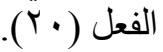

تأثير الاميدوكارب في المرور بالأمعاء الدقيقة

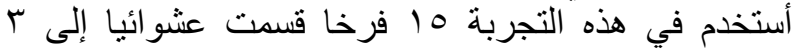

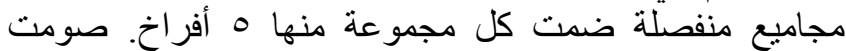

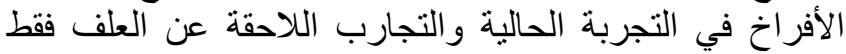

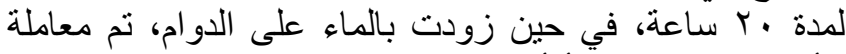

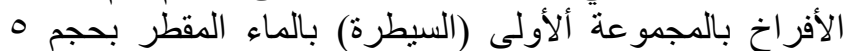
مل/كغم من وزن الجسم تحت الجلد وفي المجمو عال بالمة الثانية و الثالثة

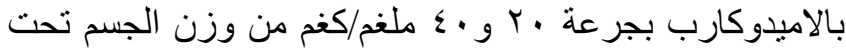

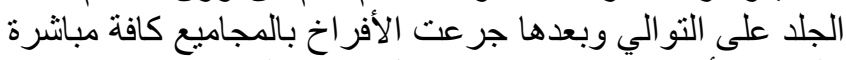

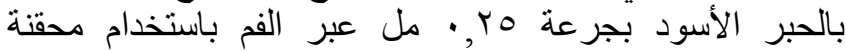

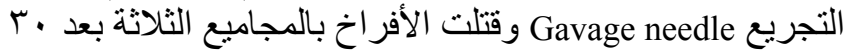

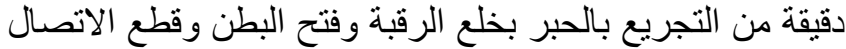

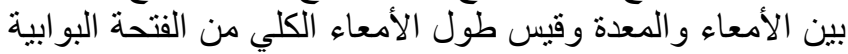
والى منطقة التقاء الأمعاء الدقيقة بالأعورين الألين

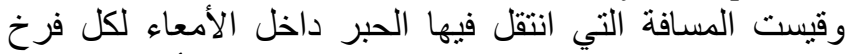

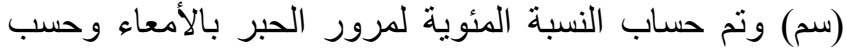

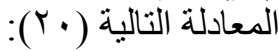

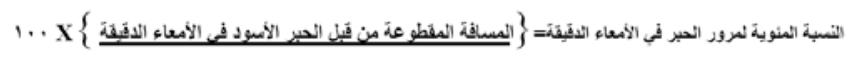
الطول الكلي للامعاء الدقيقة

قياس تأثير الديفينهايدرامين لوحده في معدل المرور في الأمعاء

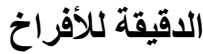

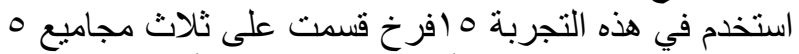

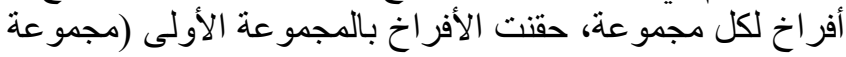
السيطرة) بالمحلول الملحي الفسلجي تحت الجلد في حين حقنت

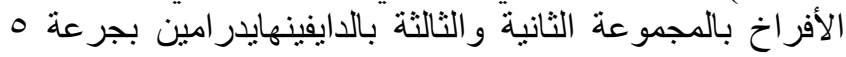

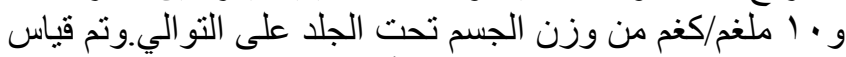

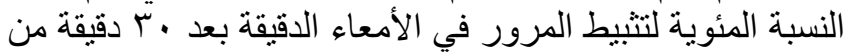
تجريع الحبر كما في المعادلة التالية (•r):

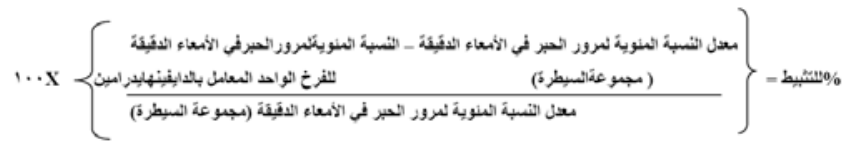

قياس تأثير الديفينهايدرامين في معدل المرور في الأمعاء

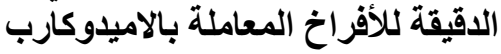

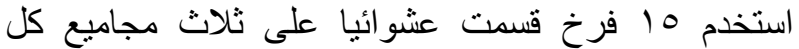

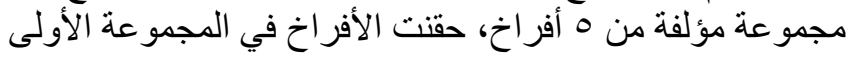

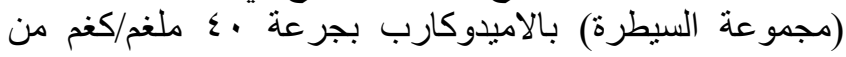




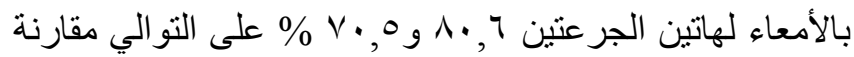

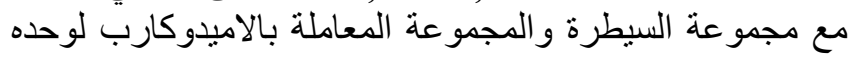

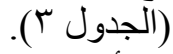

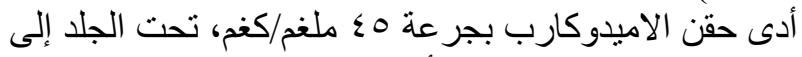

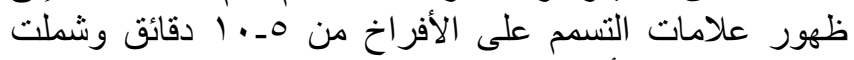

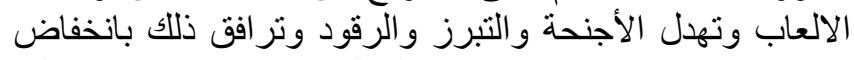

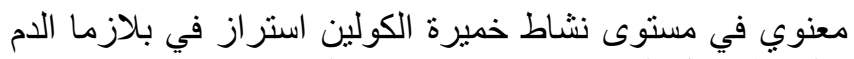

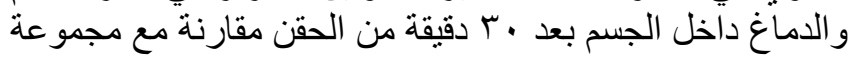

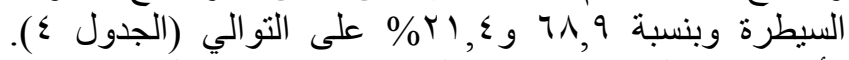

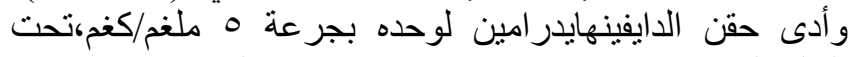

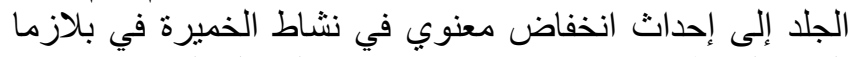

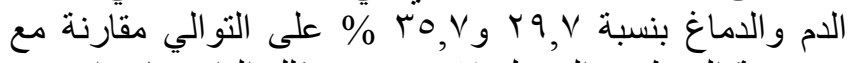

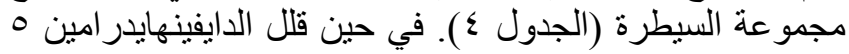

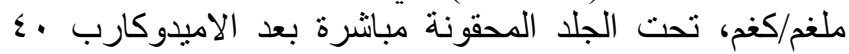

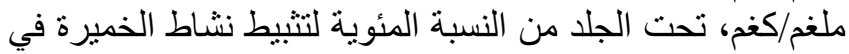

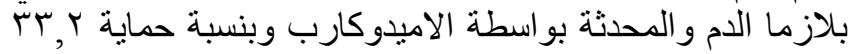
\% (الجدول ع).
من الحقن مقارنة مع مجموعة السيطرة والمجموعة المعاملة

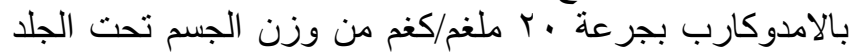

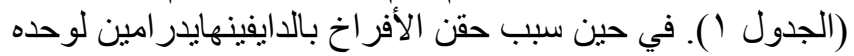

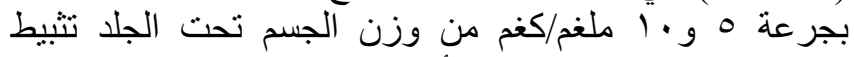

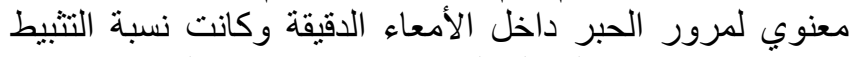

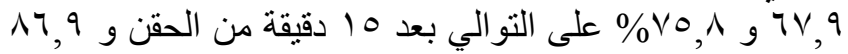

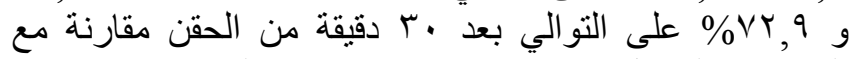

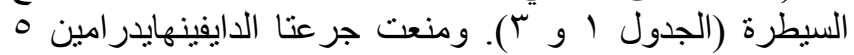

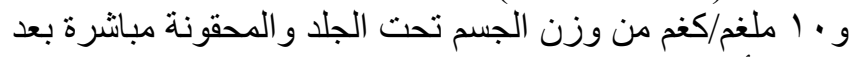

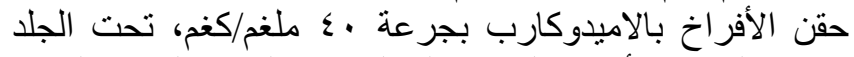

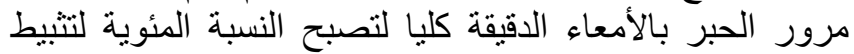

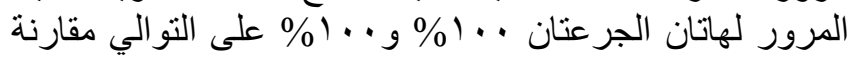

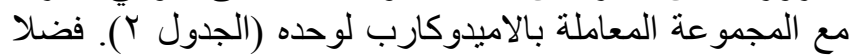

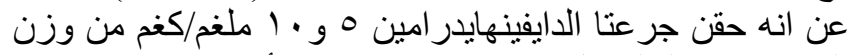

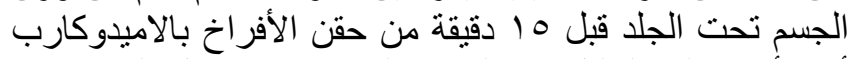

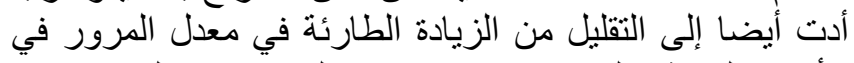

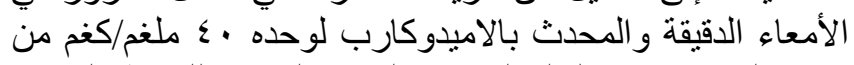

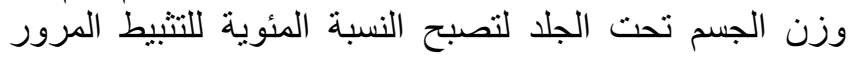

الجدول ( () تأثثر الاميدوكارب أو الدايفينهايدر امين في نسبة المرور بالأمعاء الدقيقة في أفر اخ الدجاج.

\begin{tabular}{|c|c|c|}
\hline النسبة المئوية لتثبيط المرور بالأمعاء الدقيقة & النسبة المئوية للمرور بالأمعاء الدقيقة & 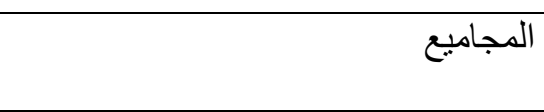 \\
\hline- & $r, T V \pm 0 \wedge, \cdot v$ & سيطرة (ماء مقطر) \\
\hline 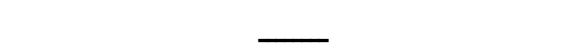 & $r, r q \pm r \varepsilon, 1 \varepsilon$ & الاميدوكارب . ب ملغم/كغم، تحت الجلد \\
\hline- & $i_{6} *, \cdots \pm i \cdots$ & الاميدوكارب · ع ملغم/كغم، تحت الجلد \\
\hline & $v, 09 \pm 0 \leqslant, q$ & سيطرة (محلول ملحي فسلجي) \\
\hline $7 \vee, 9$ & $\left.{ }^{*} \wedge, Y\right\} \pm \mid \vee, T$. & الدايفينهايدر امين م ملّغم/كغم تحت الجلد \\
\hline vo,, 1 & $*_{Y}^{*}, \Sigma 1 \pm 1 r, r$. & الدايفينهايدر امين ـ ملغخ/كغ تحت الجلد \\
\hline
\end{tabular}

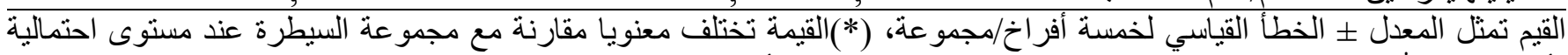

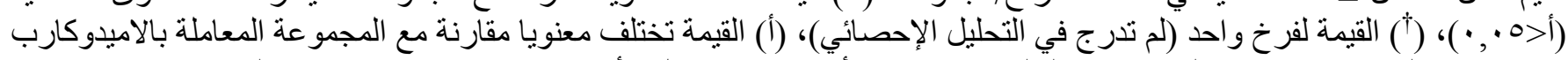

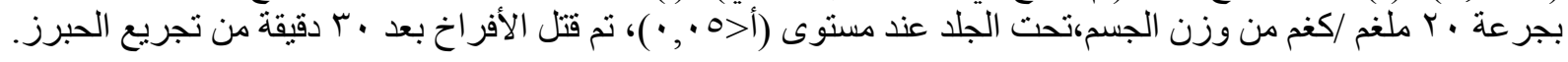

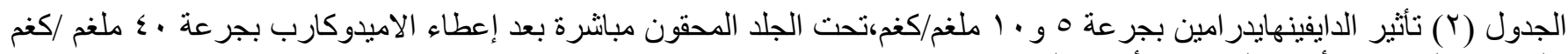
على نسبة المرور بالأمعاء الدقيقة في أفر اخخ الدجاج.

النسبة المئوية للمرور بالأمعاء النسبة المئوية لنتبيط المرور

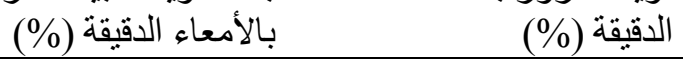

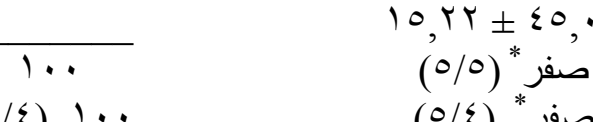

سيطرة اميدوكارب + ؛ ملغم/كغم + محلول ملحي فسلجي

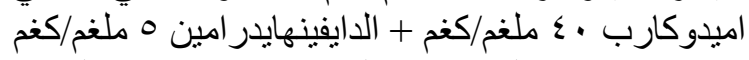

(o/乏) $1 \cdots$

صفر* (0/ )

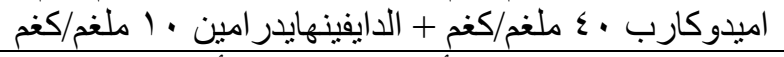

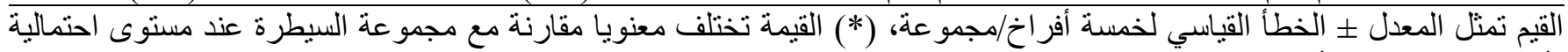

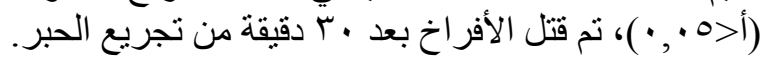




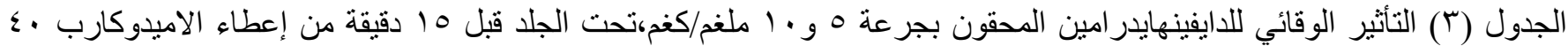

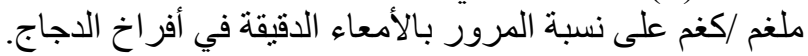

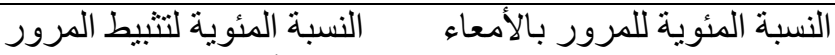

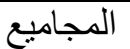
بالأمعاء الدقيقة (\%)

\author{
الاقيقة (\%)
}

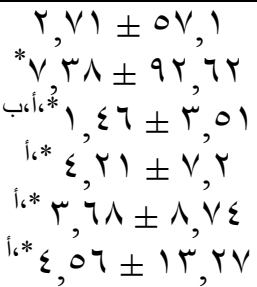

l.* $\varepsilon, 0 T \pm 1 T, Y V$

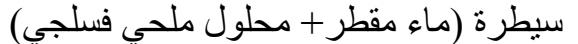

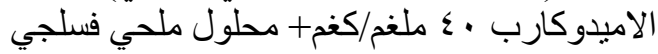

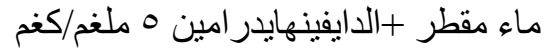

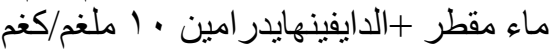

$\mathrm{VT}, \mathrm{q}$

$\Lambda \cdot, 7$

$v \cdot, 0$

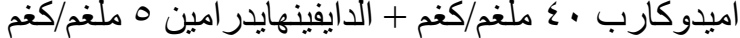

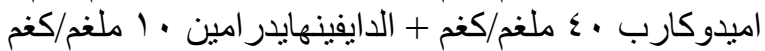

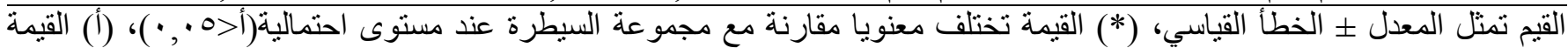

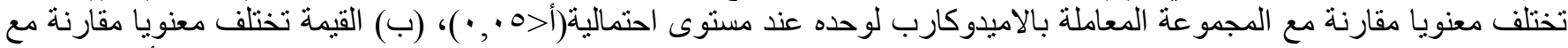

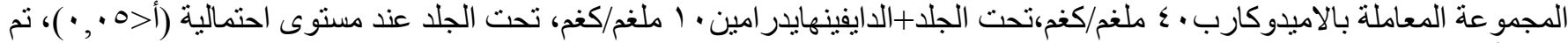
قتل الأفر اخ بعد 10 المعالمة بالائة من تجريع الحبر.

الجدول(ع) قياس تثبيط نشاط خميرة الكولين استر از في بلازما الدم و الدماغ للأفر اخ المعاملة بالاميدوكارب و الدايفينهايدر امين داخل

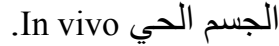

\begin{tabular}{|c|c|c|c|c|}
\hline \multicolumn{2}{|c|}{ 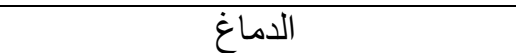 } & \multicolumn{2}{|c|}{ 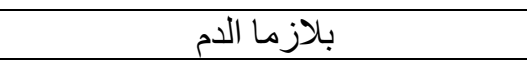 } & \multirow[b]{2}{*}{ المجاميع } \\
\hline اللنتبيط (\%) & في الباهأ/ • بـ دقيقة (التغير & 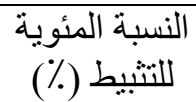 & في الباهأ/ • بـ دقيقة (التغير) & \\
\hline & $\because \cdot Y I \pm \cdot, 07$ & & $\cdot, \cdot V r_{ \pm} \cdot, v \varepsilon$ & السيطرة (ماء مقطر + محلول ملحي فسلجي) \\
\hline$\overline{r o, v}$ & ${ }^{*} \cdot, \cdot r \Delta_{ \pm} \cdot, r$ & $r q, V$ & $*, \cdot r l \pm \cdot, 0 r$ & ماء مقطر + الدايفينهايدر امينه ملغم/كغم \\
\hline Y),$\varepsilon$ & $*, \cdot \leq 0_{ \pm} \cdot, \leq \varepsilon$ & 71,9 & ${ }^{\prime * *} \cdot, r r_{ \pm} \cdot, r r$ & الاميدوكارب0؛ ملغم/كغم+ محلول ملحي فسلجي \\
\hline 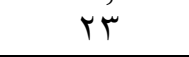 & $*, \cdot r \leqslant \pm \cdot, \leqslant \mu$ & ro,, & $\varphi^{* *} \cdot, \leqslant Y_{ \pm} \cdot, \leqslant \Lambda$ & الاميدوكارب+الدايفينهايدر امين \\
\hline
\end{tabular}

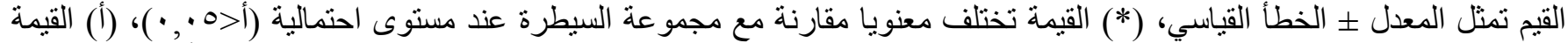

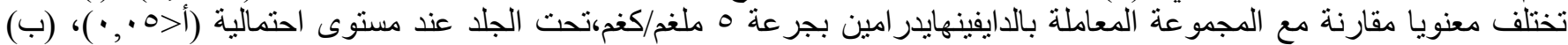

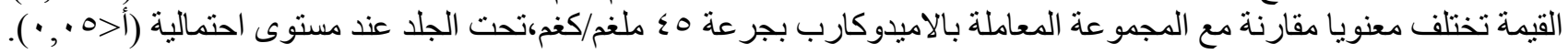

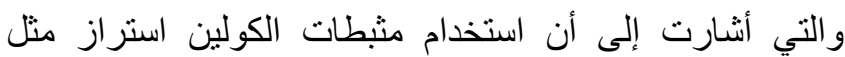

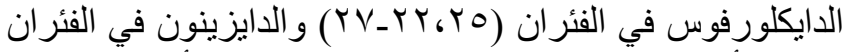

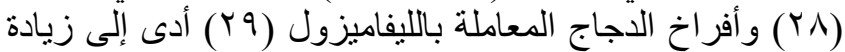

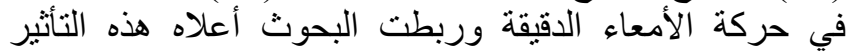

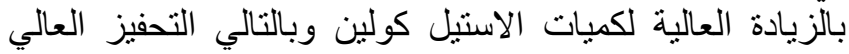

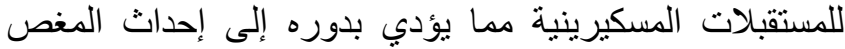

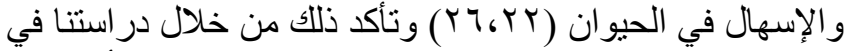

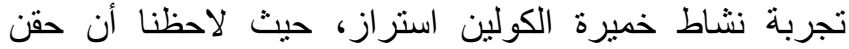

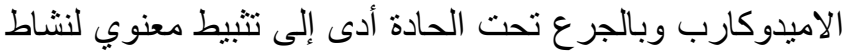

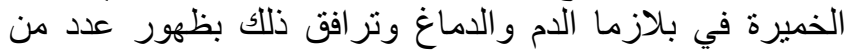

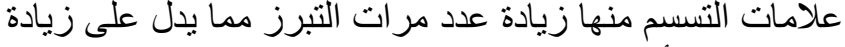

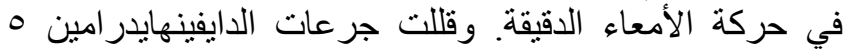

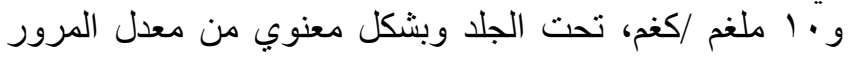

\section{المناقثة}

في دراستنا الحالية لاحظنا إن الاميدوكارب أدى إلى زيادة

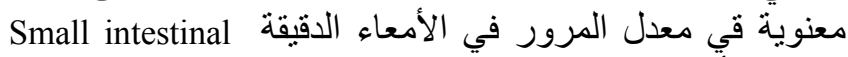

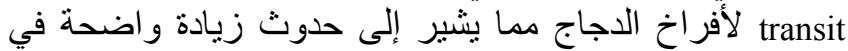

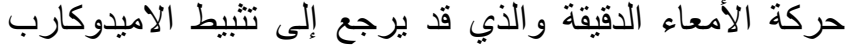

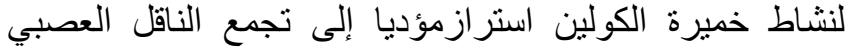

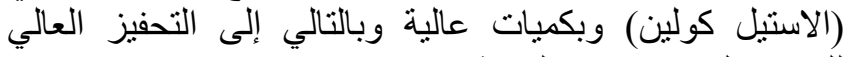
للجهاز الجنيب ودي السمبثاوي stimulation

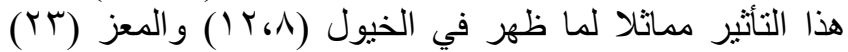

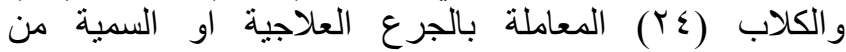
الاميدوكارب، وكانت هذه النتيجة منو افقة مع الدراسات الدات السابقة 
معنوي من النسبة المئوية لتثيط نشاط الخميرة والمحدث

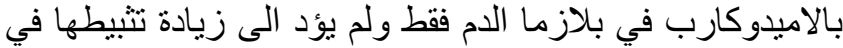

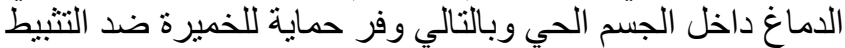

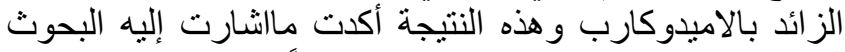

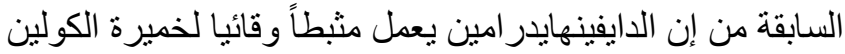

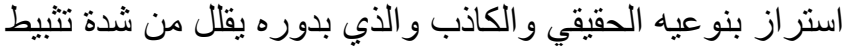

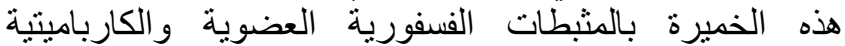

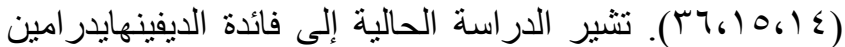

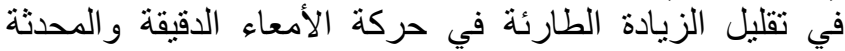

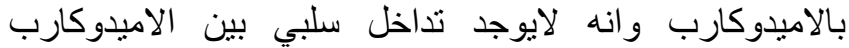
والدايفينهايدرامين على مستوى نشاط خلئ خميرة الكولين استراز

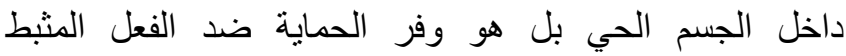
للاميدوكارب على مستوى نشاط خميرة الكولين استراز في لئي بلازما الدم ولم يؤد إلى زيادة تثبيط نشاط الخميرة في الدماغ.

الثكر والتقدير

تم دعم البحث من قبل كلية الطب البيطري، جامعة الموصل.

المصادر

1. EMEA(The European Agency of the Evaluation of Medical Products).Committee for veterinary medicinal products;2003. P.3final.

2. Katayama T, Yasuhiro H, Kazuhiro N, kyoko K, Kozo Y, Shinzo O. Imidocarb, apotant anti-protozoal up-regulates-10 production by murine acrophages. Biochemical and Biophysical Communications. 2003;2: 414-418.

3. Vial H J, Gorenflot A. Chemotherapy against babesiosis. Vet Parasitol. 2006;(138)1-2: 53 - 58.

4. Alsaad K M and AL-Mola G M. Clinical and pathological study of equine babesiosis in drought horses in Mosul. Iraqi J Vet Sci. 2006; 20(1): 89-101.

5. Kirkham W W. The treatment of equine babesiosis. Journal of American Veterinary Association. 1969;155(2): 457-460.

6. Adams H R.Veterinary pharmacology and therapeutics. $8^{\text {th }}$ ed. A Blackwell Publishing CO. Lowa State Press; 2001.pp. 1013-1014.

7. EMEA(The European Agency of the Evaluation of Medical Products). Committee for veterinary medicinal products; 2001. P.1-final.

8. Kutscha J. Effects of specific equine babesiosis treatments on equine oro-cecal transit time as measured by the lactose C-Ureide Breath Test. Thesis University of Pretoria.2008; pp.11-130.

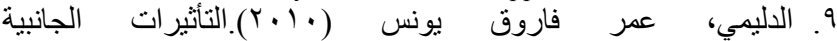

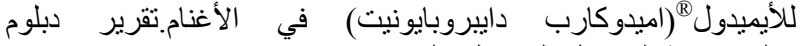

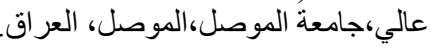

10. Tecles F and Ceron J J. Determination of whole blood cholinesterase in different animal species using specific substrates. Res. Vet. Sci. 2001; 70: 233 -238.

11. Donnellan C, Page P, Nurton P, van den Berg J S, Guthrie A. Effect of atropine and glycopyrrolate in ameliorating the side effects caused by imidocarb dipropionate administration in horses. ACVIM Abstracts, $2003 ; 454$.

12. Donnellan C. Effect of atropine and glycopyrrolate in ameliorating the clinical signs associated with the inhibition of cholinesterase activity by imidocarb diproprionate in horses. Thesis University of Pretoria. 2006; pp.14-84.

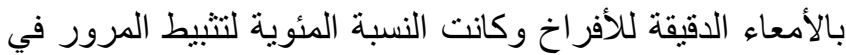

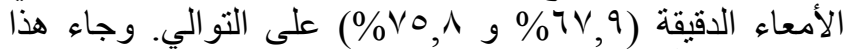

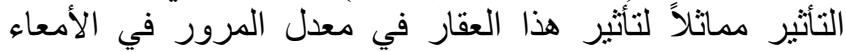

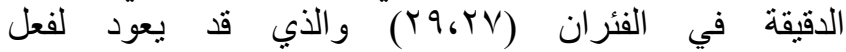

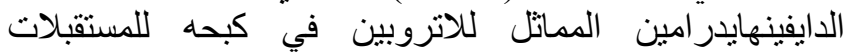

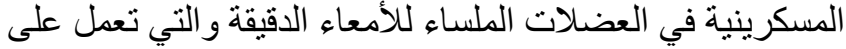

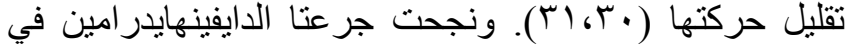
التقليل من الزيادة الطارئة في معدل المرور في في الأمعاء الدقيقة

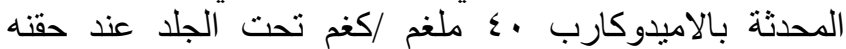

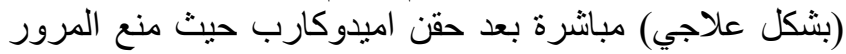

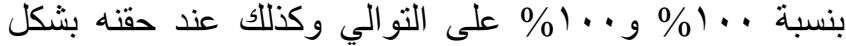

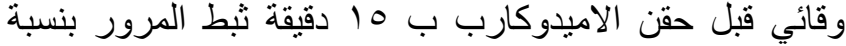

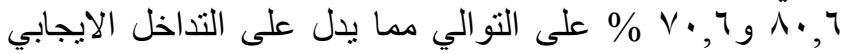

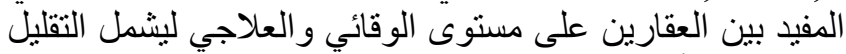

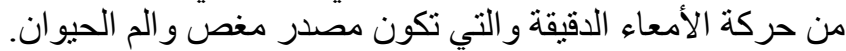

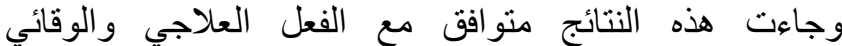
للايفينهايدر امين في التقليل من المرور في الأمعاء الدقيقة للفئران

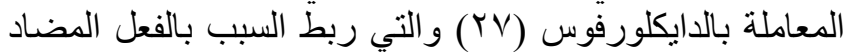

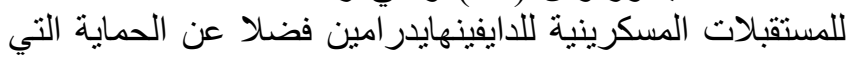

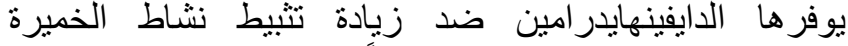

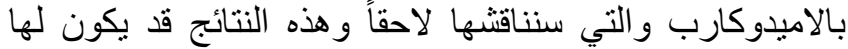

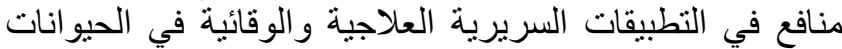

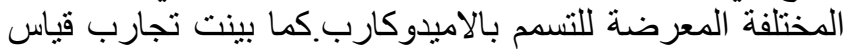

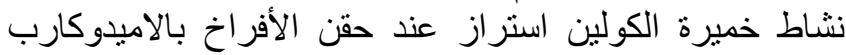

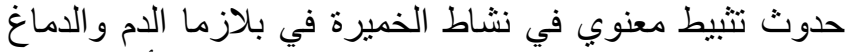

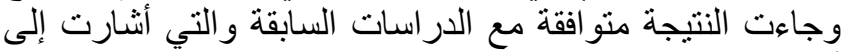

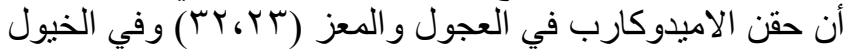

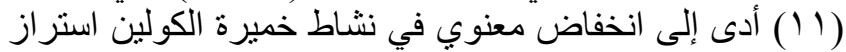

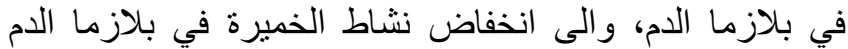

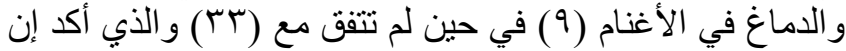

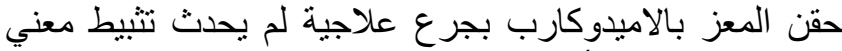

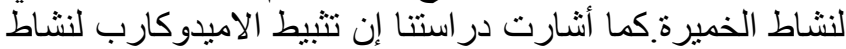

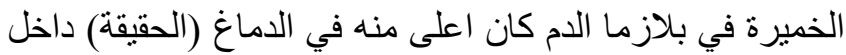

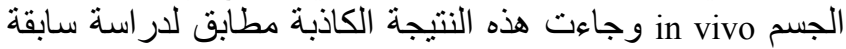

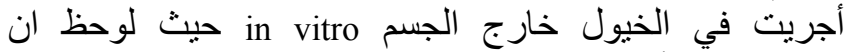
الاميدوكارب أدى إلى تثيط نشاط الخميرة الحقيقة والكاذبة (• (1)

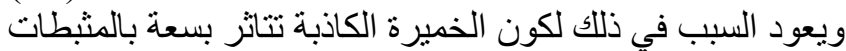

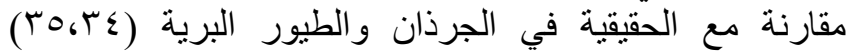

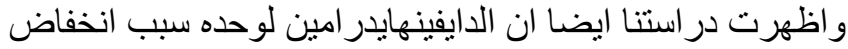

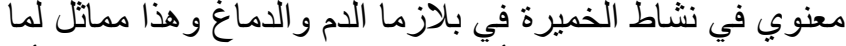

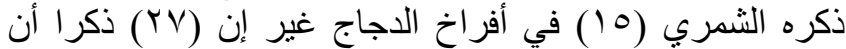

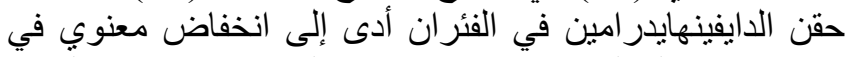

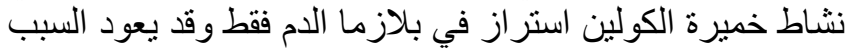

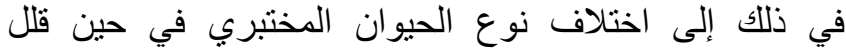
الدايفينهايدرامين المحقون مباشرة بعد الاميدوكارب الفئ وبشكل 


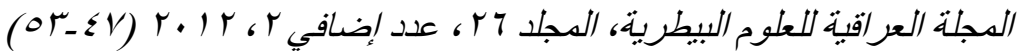 \\ وقائع الهؤتدر العلدي السادي، كلية الطب البيطري، جامعة الدوصل}

26. Taylor P. Anticholinesterase Agents. In : Gilman, A.G., Rall, T.W., Nies, A S. and Taylor P. (editors), Goodman and Gillman's The Pharmacological Basis of Therapeutic. $8^{\text {th }}$ ed., McGraw-Hill Co., Inc., New York; 1990. pp. $131-147$.

27. Faris G A M, Mohammed F K. Assessment of subtle neurobehavioral changes induced by dichlorvos in mice. Iraqi J Vet Sci. 1998; 11: 129137.

28. Yakoub L K, Mohammed F K. Organophosphorus diazinon-induced behavioral changes in mice. Iraqi J Vet Sci. 1996; 9: 15-19.

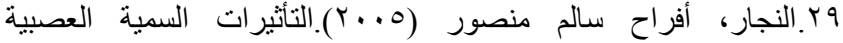

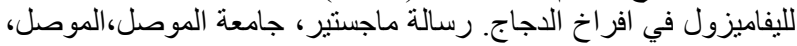

30. Pugh D M. The autonomic nervous system ,In :Brander G C, Pugh D M. Bywater R J.(Editors), Veterinary applied pharmacology and therapeutics. $4^{\text {th }}$ ed., Bailliere Tindall,London;1982.PP.75-100.

31. Douglas W W. Histamine and 5-hydroxy tryptamine (serotonin) and their antagonists. In: Gilman A G, Goodman L S, Rall T W, Murad F. (editors), Goodman and Gilman's The Pharmacological basis of therapeutics. Macmillan Publishing Co., New York, 1985.PP. 618 624.

32. Michell A R, White D G, Higgins A J, Moss P, Less P. Effect of induced hypomagnesaemia on the toxicity of imidocarb in calves. Res vet Sci. 1986; 40:264-270.

33. Singh T, varshneya C, Bahga H S, Sharma L D. Adverse effect of Imidocarb dipropionate administration in goats.1990;40(2-3);119-127.

34. Mohammed F K, Faris G A M, Shindala M K h. Comparative antidotal effects of diphenhydramine and atropine against dichlorvos induced acute toxicosis in rats. Vet Arhiv. 2002; 72 (1) : $19-28$.

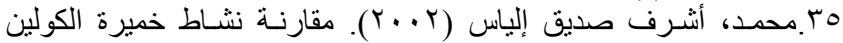

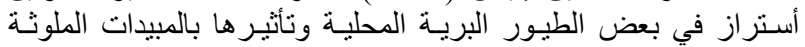

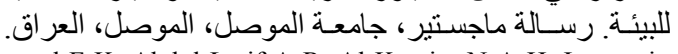

36. Mohammed F K, Abdul-Latif A R, Al-Kassim N A H. Interaction of diphenhydramine with cholinesterase inhibitors in mice. Toxicol Lett. $1987 ; 37: 235-240$.
13. Fikes J D. Organophosphorus and carbamate insecticides. Vet Clin North Am Small Anim Prac. 1990; 20 (2) : 353 - 367.

14. Taylor P, Kovarik Z, Reiner E, Radic Z. Acetylcholinesterase: Converting a vulnerable target to a template for antidotes and detection of inhibitor exposure. Toxicol. 2007; $233: 70$ - 78.

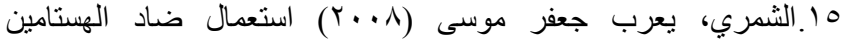

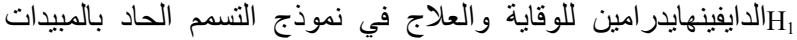

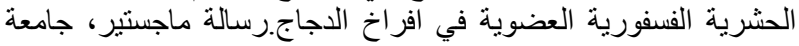

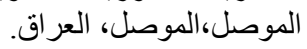

17

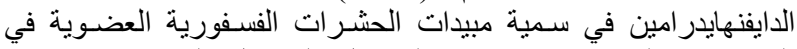
الفئر ان. رسـالة ماجستير، في جامعة الموصل، الموصل، العراق.

17. Coles E H. Veterinary clinical pathology, $4^{\text {th }}$ edition, W.B. Saunders CO. Philadelphia. London. Toronto; 1986. pp.48-55.

18. Mohammed F K, St.Omer V E V.Modifications of Michel's electrometric method for rapid measurement of blood cholinesterase activity in animals:a mini review.Vet Hum Toxicol. 1982;24(2):119121.

19. Mohammed F K, Alias A S, and Ahmed OAH. Electrometric measurement of plasma, erythrocyte and whole blood cholinesterase activities in healthy Human volunteers. J Med Toxicol. 2007a ; 3 (1) : $25-30$.

20. Hsu W H. Xylazine-induced delay of small intestinal transit in mice. Eur. J. Pharmacol. 1982; 83: 55-60.

21. Petrie A, Watson P. Statistics for Veterinary and Animal Sciences. Blackwell Science, Oxford ; 1999. pp. 90 - 140.

22. Hayes W J. Pesticides studied in man. Willams and Wilkins, Baltimore, U.S.A.;1982.PP.284-413.

23. Ali B H, Hassan T, Suliman H B, Abdelsalam A B. Some effects of imidocarb in goats. Vet Hum Toxicol. 1985; 27:477-480.

24. Abdullah A S, Sheikh-Omar A R, Baggot J D, Zamari M. Adverse effects of imidocarb dipropionate (imizol) in dog. Vet Res Com. 1984; 8:55-59.

25. Osweiler G D, Carson T L, Buck W B, Van-Gelder G A. Clinical and diagnostic veterinary toxicology. 3rd ed., Kendall Hunt Publishing Co., Dubuque. Iowa; 1985. pp. 298-317. 